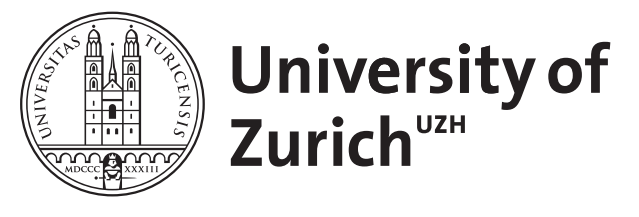

\title{
Functional-type a posteriori error estimates for mixed finite element methods
}

\author{
Repin, S ; Smolianski, A
}

\begin{abstract}
This paper concerns a posteriori error estimation for the primal and dual mixed finite element methods applied to the diffusion problem. The problem is considered in a general setting with inhomogeneous mixed Dirichlet-Neumann boundary conditions. New functional-type a posteriori error estimators are proposed that exhibit the ability both to indicate the local error distribution and to ensure upper bounds for discretization errors in primal and dual (flux) variables. The latter property is a direct consequence of the absence in the estimators of any mesh-dependent constants; the only constants present in the estimates stem from the Friedrichs and trace inequalities and, thus, are global and dependent solely on the domain geometry and the bounds of the diffusion matrix. The estimators are computationally cheap and require only the projections of piecewise constant functions onto the spaces of the lowest-order Raviart-Thomas or continuous piecewise linear elements. It is shown how these projections can be easily realized by simple local averaging.
\end{abstract}

DOI: https://doi.org/10.1515/156939805775122271

Posted at the Zurich Open Repository and Archive, University of Zurich

ZORA URL: https://doi.org/10.5167/uzh-21739

Journal Article

Published Version

Originally published at:

Repin, S; Smolianski, A (2005). Functional-type a posteriori error estimates for mixed finite element methods. Russian Journal of Numerical Analysis and Mathematical Modelling, 20(4):365-382.

DOI: https://doi.org/10.1515/156939805775122271 
Russ. J. Numer. Anal. Math. Modelling, Vol. 20, No. 4, pp. 365-382 (2005)

(c) VSP 2005

\title{
Functional-type a posteriori error estimates for mixed finite element methods
}

\author{
S. I. REPIN* and A. SMOLIANSKI ${ }^{\dagger}$
}

\begin{abstract}
This paper concerns a posteriori error estimation for the primal and dual mixed finite element methods applied to the diffusion problem. The problem is considered in a general setting with inhomogeneous mixed Dirichlet-Neumann boundary conditions. New functional-type a posteriori error estimators are proposed that exhibit the ability both to indicate the local error distribution and to ensure upper bounds for discretization errors in primal and dual (flux) variables. The latter property is a direct consequence of the absence in the estimators of any mesh-dependent constants; the only constants present in the estimates stem from the Friedrichs and trace inequalities and, thus, are global and dependent solely on the domain geometry and the bounds of the diffusion matrix. The estimators are computationally cheap and require only the projections of piecewise constant functions onto the spaces of the lowest-order Raviart-Thomas or continuous piecewise linear elements. It is shown how these projections can be easily realized by simple local averaging.
\end{abstract}

This paper is dedicated to the anniversary of Prof. Yu. A. Kuznetsov

Mixed finite element methods are frequently used for solving problems of computational mechanics, especially when one needs accurate and conserving approximations for fluxes and stresses. These variables are of vital importance in many problems of heat conduction, mass diffusion, electrostatics, elasticity and flow in porous media, which generates permanently growing interest in the development and application of mixed methods. Recently, the tight link between the mixed and finite volume methods have been emphasized in [1,21,23]; for distorted polygonal and polyhedral meshes a new version of mixed finite element approximation has been presented in [13].

The fundamental analysis of mixed formulations and a priori estimates for the mixed finite element methods can be found in $[6,12,20]$. However, for practical applications, a posteriori error estimates seem even more important, as they should provide both guidelines for an adaptive improvement of the approximation and a stopping criterion for the computational process. Although this ultimate goal of the a posteriori error estimation was not always achieved, many different estimators were proposed for mixed finite elements. Residual-based estimates have been derived in $[1,2,4,7,11]$ for a diffusion-type equation and extended in $[9,14]$ to the

\footnotetext{
${ }^{*}$ V. A. Steklov Institute of Mathematics, Fontanka 27, 191011 St. Petersburg, Russia; E-mail: repin@pdmi.ras.ru

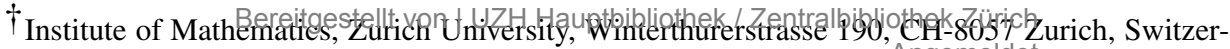
land; E-mail: antsmol@amath.unizh.ch 
equations of linear elasticity. The superconvergence-based (averaging-type) error estimators are proposed in $[5,8]$ to control the $L_{2}$-error of the flux variable. Further, the estimators based on the solution of local problems are given in $[2,11,14]$ and a hierarchical estimator can be found in [22]. Finally, a comparison of these four types of error estimators for mixed finite element discretizations by Raviart-Thomas elements is presented in [22].

In this paper we apply a posteriori error estimator of another type, the so-called functional-type estimator (see [16-19]) to the approximations obtained by the primal and dual mixed finite element methods. While the proposed estimator can be easily used for the indication of local error distribution, its main distinctive feature and strength is the absence of any discretization-dependent constants; the only constants present in the estimator stem from the Friedrichs and trace inequalities, they depend solely on the domain geometry and the known bounds of the diffusion matrix and can be readily evaluated just once before the beginning of the computation. That is why the estimator provides a guaranteed upper bound for the error and can be utilized as a stopping criterion in the adaptive approximation.

It should be noted that in this work we restrict ourselves to the consideration of a classical diffusion problem. However, the suggested method can be applied to other boundary value problems, where the form of functional-type error estimates is known. The rest of the paper is organized as follows. In Section 2, we introduce the notation and discuss the primal and dual mixed formulations of the diffusion problem with inhomogeneous mixed Dirichlet-Neumann boundary conditions. Although both formulations have been addressed in many different works, a thorough derivation for the case of nonhomogeneous mixed boundary conditions does not seem commonplace and is presented here for the sake of completeness. In Section 3, the a posteriori error estimator for the primal mixed method is derived, whilst Section 4 is devoted to the a posteriori estimation of the dual mixed finite element method based on the Raviart-Thomas space.

\section{NOTATION AND BASIC RELATIONS}

We consider the diffusion problem

$$
\begin{aligned}
\operatorname{div} \mathbf{A} \nabla u+f & =0 & & \text { in } \Omega \\
u & =u_{0} & & \text { on } \partial_{1} \Omega \\
\mathbf{A} \nabla u \cdot \mathbf{n} & =F & & \text { on } \partial_{2} \Omega
\end{aligned}
$$

where $\Omega$ is a bounded domain in $\mathbb{R}^{d}, d=2,3$, with a Lipschitz continuous boundary $\partial \Omega$ that consists of two non-intersecting parts $\partial_{1} \Omega$ and $\partial_{2} \Omega$ and $\mathbf{n}$ is the outward normal to $\partial \Omega$. We assume that meas $\left(\partial_{1} \Omega\right) \neq 0$.

Further, by letters $u, v, w$ we denote the functions associated with the solution to the problem (the primal variable), by letters $\mathbf{p}, \mathbf{q}, \mathbf{y}, \eta$ the vector-valued functions

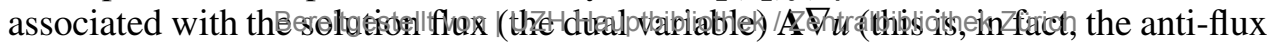
because of the absence of the minus sign, but we still call it theneffildet for brevity). 
Also, we assume that $\mathbf{A}$ is a symmetric matrix with components from $L_{\infty}(\Omega)$ that satisfies the following condition with certain positive constants $c_{1}$ and $c_{2}$ :

$$
c_{1}|\xi|^{2} \leqslant \mathbf{A}(x) \xi \cdot \xi \leqslant c_{2}|\xi|^{2} \quad \forall \xi \in \mathbb{R}^{d} \text {, for a.e. } x \in \Omega
$$

where $|\xi|=(\xi \cdot \xi)^{1 / 2}$ and the dot denotes the scalar product of vectors in $\mathbb{R}^{d}$.

As to the problem data, we suppose that

$$
u_{0} \in H^{1}(\Omega), \quad f \in L_{2}(\Omega), \quad F \in L_{2}\left(\partial_{2} \Omega\right) .
$$

The following functional spaces will be frequently used throughout the paper:

$$
\begin{gathered}
V:=H^{1}(\Omega), \quad V_{0}:=\left\{v \in V \mid v=0 \text { on } \partial_{1} \Omega\right\}, \quad Q:=L_{2}\left(\Omega ; \mathbb{R}^{d}\right) \\
\widehat{V}:=L_{2}(\Omega), \quad \widehat{Q}:=H(\Omega ; \operatorname{div}), \quad \widehat{Q}^{+}:=\left\{\mathbf{y} \in \widehat{Q}|\mathbf{y} \cdot \mathbf{n}|_{\partial_{2} \Omega} \in L_{2}\left(\partial_{2} \Omega\right)\right\} .
\end{gathered}
$$

Here ' $V$ ' correspond to the spaces for the primal variable $u$, ' $Q$ ' to the spaces for the dual variable $\mathbf{p}$, the spaces without 'hats' are used for the primal mixed method, while the spaces with 'hats' for the dual mixed one.

Further, by $\|\cdot\|$ and $\|\cdot\|_{\partial_{2} \Omega}$ we denote the $L_{2}$-norm on $\Omega$ and on $\partial_{2} \Omega$, respectively, by $\|\cdot\|_{1}$ the $H^{1}$-norm on $\Omega$ and by $\|\cdot\|_{\text {div }}$ the norm in $H(\Omega$; div $)$ $\left(\|\cdot\|_{\text {div }}:=\left(\|\cdot\|^{2}+\|\operatorname{div} \cdot\|^{2}\right)^{1 / 2}\right)$; we also define $\|\mathbf{q}\|:=\left(\int_{\Omega} \mathbf{A q} \cdot \mathbf{q} \mathrm{d} x\right)^{1 / 2}$ and $\|\mathbf{q}\|_{*}:=\left(\int_{\Omega} \mathbf{A}^{-1} \mathbf{q} \cdot \mathbf{q} \mathrm{d} x\right)^{1 / 2}$ for all $\mathbf{q} \in Q$.

In the a posteriori estimates we also use the functional

$$
D(\nabla v, \mathbf{y}):=\int_{\Omega}\left(\frac{1}{2} \mathbf{A} \nabla v \cdot \nabla v+\frac{1}{2} \mathbf{A}^{-1} \mathbf{y} \cdot \mathbf{y}-\nabla v \cdot \mathbf{y}\right) \mathrm{d} x=\frac{1}{2}\|\mathbf{A} \nabla v-\mathbf{y}\|_{*}^{2}
$$

that is defined for all $v \in V$ and $\mathbf{y} \in Q$.

It is well known (see, e.g., $[6,10])$ that the generalized solution of problem (1.1)-(1.3) can be viewed as a saddle point of the Lagrangian

$$
L(v, \mathbf{q}):=\int_{\Omega}\left(\nabla v \cdot \mathbf{q}-\frac{1}{2} \mathbf{A}^{-1} \mathbf{q} \cdot \mathbf{q}\right) \mathrm{d} x-l(v)
$$

where

$$
l(v)=\int_{\Omega} f v \mathrm{~d} x+\int_{\partial_{2} \Omega} F v \mathrm{~d} s .
$$

The saddle point $(u, \mathbf{p})$ is sought on the pair of sets $\left(V_{0}+u_{0}\right) \times Q$ and satisfies the conditions

$$
\int_{\Omega}\left(\mathbf{A}^{-1} \mathbf{p}-\nabla u\right) \cdot \mathbf{q} \mathrm{d} x=0 \quad \forall \mathbf{q} \in Q
$$


In this formulation the condition $\mathbf{p}=\mathbf{A} \nabla u$ is satisfied in the $L_{2}$-sense and (1.7) shows that the relations $\operatorname{div} \mathbf{p}+f=0$ in $\Omega$ and $\mathbf{p} \cdot \mathbf{n}=F$ on $\partial_{2} \Omega$ are satisfied in a weak sense.

The Lagrangian $L$ generates two functionals

$$
J(v):=\sup _{\mathbf{q} \in Q} L(v, \mathbf{q})=\frac{1}{2}\|\nabla v\|^{2}-l(v)
$$

and

$$
I^{*}(\mathbf{q}):=-\frac{1}{2}\|\mathbf{q}\|_{*}^{2}-l\left(u_{0}\right)+\int_{\Omega} \nabla u_{0} \cdot \mathbf{q} \mathrm{d} x .
$$

It is well known (see [10]) that

$$
\inf _{v \in V_{0}+u_{0}} J(v)=: \inf \mathscr{P}=\sup \mathscr{P}^{*}:=\sup _{\mathbf{q} \in Q_{l}} I^{*}(\mathbf{q}) .
$$

Here

$$
Q_{l}:=\left\{\mathbf{q} \in Q \mid \int_{\Omega} \mathbf{q} \cdot \nabla w \mathrm{~d} x=l(w) \forall w \in V_{0}\right\} .
$$

By standard arguments, one can prove that the unique saddle point $(u, \mathbf{p})$ exists and satisfies the relation

$$
L(u, \mathbf{p})=\inf \mathscr{P}=\sup \mathscr{P}^{*} .
$$

If $Q_{h} \subset Q$ and $V_{0 h} \subset V_{0}$ are certain subspaces constructed by finite element approximation, then the discrete analog of (1.6)-(1.7) is the primal mixed finite element $\operatorname{method}$ (see [20]): Find $\left(u_{h}, \mathbf{p}_{h}\right) \in\left(V_{0 h}+u_{0}\right) \times Q_{h}$ such that

$$
\begin{aligned}
\int_{\Omega}\left(\mathbf{A}^{-1} \mathbf{p}_{h}-\nabla u_{h}\right) \cdot \mathbf{q}_{h} \mathrm{~d} x=0 & \forall \mathbf{q}_{h} \in Q_{h} \\
\int_{\Omega} \mathbf{p}_{h} \cdot \nabla w_{h} \mathrm{~d} x-l\left(w_{h}\right)=0 & \forall w_{h} \in V_{0 h} .
\end{aligned}
$$

Another mixed formulation arises if we rewrite the Lagrangian $L$ in a somewhat different form. First, we introduce the functional $g:\left(V_{0}+u_{0}\right) \times \widehat{Q} \rightarrow \mathbb{R}$ by the relation

$$
g(v, \mathbf{q}):=\int_{\Omega}(\nabla v \cdot \mathbf{q}+v(\operatorname{div} \mathbf{q})) \mathrm{d} x .
$$

We have

$$
L(v, \mathbf{q})=-\frac{1}{2}\|\mathbf{q}\|_{*}^{2}-\int_{\Omega} v(\operatorname{div} \mathbf{q}) \mathrm{d} x-l(v)+g(v, \mathbf{q}) .
$$

We introduce the set

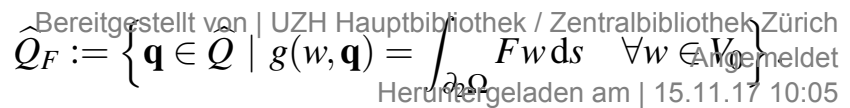


Note that for $\mathbf{q} \in \widehat{Q}_{F}$ we have

$$
g(v, \mathbf{q})=g\left(w+u_{0}, \mathbf{q}\right)=g(w, \mathbf{q})+g\left(u_{0}, \mathbf{q}\right)=\int_{\partial_{2} \Omega} F w \mathrm{~d} s+g\left(u_{0}, \mathbf{q}\right) \forall w \in V_{0} .
$$

Therefore, if the variable $\mathbf{q}$ is taken not from $Q$ but from the narrower set $\widehat{Q}_{F}$, then the Lagrangian can be written as

$$
\widehat{L}(v, \mathbf{q}):=-\frac{1}{2}\|\mathbf{q}\|_{*}^{2}-\int_{\Omega} v(\operatorname{div} \mathbf{q}) \mathrm{d} x-\int_{\Omega} f v \mathrm{~d} x-\int_{\partial_{2} \Omega} F u_{0} \mathrm{~d} s+g\left(u_{0}, \mathbf{q}\right) .
$$

We see that the Lagrangian $\widehat{L}$ is defined on a wider set of primal functions: here $v \in \widehat{V}$.

The problem of finding $(\widehat{u}, \widehat{\mathbf{p}}) \in \widehat{V} \times \widehat{Q}_{F}$ such that

$$
\widehat{L}(\widehat{u}, \widehat{\mathbf{q}}) \leqslant \widehat{L}(\widehat{u}, \widehat{\mathbf{p}}) \leqslant \widehat{L}(\widehat{v}, \widehat{\mathbf{p}}) \quad \forall \widehat{\mathbf{q}} \in \widehat{Q}_{F}, \forall \widehat{v} \in \widehat{V}
$$

is known as the dual mixed formulation of problem (1.1)-(1.3) (see, e.g. [6]).

From (1.13) the necessary conditions can be derived:

$$
\begin{aligned}
\int_{\Omega}\left(\mathbf{A}^{-1} \widehat{\mathbf{p}} \cdot \widehat{\mathbf{q}}+(\operatorname{div} \widehat{\mathbf{q}}) \widehat{u}\right) \mathrm{d} x & =g\left(u_{0}, \widehat{\mathbf{q}}\right) \quad \forall \widehat{\mathbf{q}} \in \widehat{Q}_{0} \\
\int_{\Omega}(\operatorname{div} \widehat{\mathbf{p}}+f) \widehat{v} \mathrm{~d} x & =0 \quad \forall \widehat{v} \in \widehat{V} .
\end{aligned}
$$

Note that the condition $\operatorname{div} \widehat{\mathbf{p}}+f=0$ is satisfied in a 'strong' (i.e. in $L_{2}$ ) sense, the Neumann type boundary condition (1.3) is regarded as an essential boundary condition, the relation $\widehat{\mathbf{p}}=\mathbf{A} \nabla \widehat{u}$ and the Dirichlet-type boundary condition (1.2) are satisfied in a weak sense.

The Lagrangian $\widehat{L}$ also generates two functionals

$$
\widehat{J}(\widehat{v}):=\sup _{\widehat{\mathbf{q}} \in \widehat{Q}_{F}} \widehat{L}(\widehat{v}, \widehat{\mathbf{q}}), \quad \widehat{I}^{*}(\widehat{\mathbf{q}}):=\inf _{\widehat{v} \in \widehat{V}} \widehat{L}(\widehat{v}, \widehat{\mathbf{q}}) .
$$

The two corresponding variational problems are $\inf _{\widehat{v} \in \widehat{V}} \widehat{J}(\widehat{v})$ and $\sup _{\widehat{\mathbf{q}} \in \widehat{Q}_{F}} \widehat{I}^{*}(\widehat{\mathbf{q}})$. They are called Problems $\widehat{\mathscr{P}}$ and $\widehat{\mathscr{P}}^{*}$, respectively. Note that the functional $\widehat{J}$ (unlike $J$ ) has no simple explicit form. However, we can prove the solvability of Problem $\widehat{\mathscr{P}}$ by the following Lemma.

Lemma 1.1. For any $\widehat{v} \in \widehat{V}$ and $F \in L_{2}\left(\partial_{2} \Omega\right)$, there exists $\mathbf{p}^{v} \in \widehat{Q}_{F}$ such that

$$
\operatorname{div} \mathbf{p}^{v}+\widehat{v}=0 \quad \text { in } \Omega
$$

Bereitgestellt von $\left\|\mathbf{p}^{v}\right\|_{* Z} \leqslant C_{\Omega}\left(\|\widehat{v}\|+\|F\| \|_{\partial_{2} \Omega}\right)$. 
Proof. We know that the boundary value problem

$$
\begin{aligned}
\operatorname{div} \mathbf{A} \nabla u^{v}+\widehat{v} & =0 & & \text { in } \Omega \\
u^{v} & =0 & & \text { on } \partial_{1} \Omega \\
\mathbf{A} \nabla u^{v} \cdot \mathbf{n} & =F & & \text { on } \partial_{2} \Omega
\end{aligned}
$$

possesses the unique solution $u^{v} \in V_{0}$, and the energy estimate

$$
\left\|\nabla u^{v}\right\| \leqslant C_{\Omega}\left(\|\widehat{v}\|+\|F\|_{\partial_{2} \Omega}\right)
$$

holds. Let $\mathbf{p}^{v}:=\mathbf{A} \nabla u^{v}$. Obviously, $\mathbf{p}^{v} \in \widehat{Q}_{F}$ and, since

$$
\left\|\mathbf{p}^{v}\right\|_{*}^{2}=\int_{\Omega} \mathbf{A}^{-1}\left(\mathbf{A} \nabla u^{v}\right) \cdot\left(\mathbf{A} \nabla u^{v}\right) \mathrm{d} x=\left\|\nabla u^{v}\right\|^{2}
$$

we find that (1.17) holds. From (1.18) we also see that (1.16) is valid.

By Lemma 1.1 we can easily prove the coercivity of $\widehat{J}$ on $\widehat{V}$. Indeed,

$$
\begin{aligned}
\widehat{J}(\widehat{v}) \geqslant \widehat{L}\left(\widehat{v}, \alpha \mathbf{p}^{v}\right)= & -\frac{1}{2} \alpha^{2}\left\|\mathbf{p}^{v}\right\|_{*}^{2}+\alpha\|\widehat{v}\|^{2} \\
& -\|f\|\|\widehat{v}\|+g\left(u_{0}, \alpha \mathbf{p}^{v}\right)-\int_{\partial_{2} \Omega} F u_{0} \mathrm{~d} s .
\end{aligned}
$$

Here $\left|g\left(u_{0}, \alpha \mathbf{p}^{v}\right)\right| \leqslant \alpha\left\|\mathbf{p}^{v}\right\|_{\operatorname{div}}\left\|u_{0}\right\|_{1}$ and

$$
\begin{aligned}
\left\|\mathbf{p}^{v}\right\|_{\text {div }}^{2} & =\left\|\mathbf{p}^{v}\right\|^{2}+\left\|\operatorname{div} \mathbf{p}^{v}\right\|^{2} \leqslant \frac{1}{c_{1}}\left\|\mathbf{p}^{v}\right\|_{*}^{2}+\|\widehat{v}\|^{2} \\
& \leqslant \frac{1}{c_{1}} C_{\Omega}^{2}\left(\|\widehat{v}\|+\|F\|_{\partial_{2} \Omega}\right)^{2}+\|\widehat{v}\|^{2} .
\end{aligned}
$$

Therefore,

$$
\widehat{J}(\widehat{v}) \geqslant-\frac{1}{2} \alpha^{2} C_{\Omega}^{2}\|\widehat{v}\|^{2}+\alpha\|\widehat{v}\|^{2}+\Theta(\|\widehat{v}\|)+\Theta_{0}
$$

where $\Theta(\|\widehat{v}\|)$ contains the terms linear with respect to $\|\widehat{v}\|$ and $\Theta_{0}$ does not depend on $\widehat{v}$. Take $\alpha=1 / C_{\Omega}^{2}$. Then

$$
\widehat{J}(\widehat{v}) \geqslant \frac{1}{2 C_{\Omega}^{2}}\|\widehat{v}\|^{2}+\Theta(\|\widehat{v}\|)+\Theta_{0} \longrightarrow+\infty \quad \text { as }\|\widehat{v}\| \rightarrow \infty .
$$

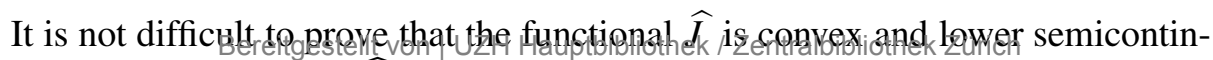
uous. Therefore, Problem $\mathscr{P}$ has a solution $\widehat{u}$. 
Remark 1.1. Lemma 1.1 implies the inf-sup condition

$$
\inf _{\substack{\varphi \in L_{2}(\Omega) \\ \psi \in L_{2}\left(\partial_{2} \Omega\right)}} \sup _{\mathbf{q} \in \widehat{Q}_{F}} \frac{\int_{\Omega} \varphi \operatorname{div} \mathbf{q} \mathrm{d} x+\int_{\partial_{2} \Omega} \psi \mathbf{q} \cdot \mathbf{n} \mathrm{d} s}{\|\mathbf{q}\|_{\operatorname{div}}\left(\|\varphi\|^{2}+\|\psi\|_{\partial_{2} \Omega}^{2}\right)^{1 / 2}} \geqslant C_{0}>0 .
$$

Now consider the functional $\widehat{I^{*}}$. We have $\widehat{I^{*}}(\widehat{\mathbf{q}})=-\frac{1}{2}\|\widehat{\mathbf{q}}\| \|_{*}^{2}+g\left(u_{0}, \widehat{\mathbf{q}}\right)-\int_{\partial_{2} \Omega} F u_{0} \mathrm{~d} s$, if $\operatorname{div} \widehat{\mathbf{q}}+f=0$ in $L_{2}(\Omega)$, and $\widehat{I}^{*}(\widehat{\mathbf{q}})=-\infty$ in all other cases. Since $\widehat{\mathbf{q}} \in \widehat{Q}_{F}$, we have

$$
\int_{\Omega} \nabla w \cdot \widehat{\mathbf{q}} \mathrm{d} x=-\int_{\Omega}(\operatorname{div} \widehat{\mathbf{q}}) w \mathrm{~d} x+\int_{\partial_{2} \Omega} F w \mathrm{~d} s \quad \forall w \in V_{0} .
$$

Recalling that $\operatorname{div} \widehat{\mathbf{q}}=-f$ in $L_{2}(\Omega)$, we find that the dual functional for such a case has the form

$$
\begin{aligned}
-\frac{1}{2}\|\widehat{\mathbf{q}}\|_{*}^{2} & +\int_{\Omega}\left(\nabla u_{0} \cdot \widehat{\mathbf{q}}-f u_{0}\right) \mathrm{d} x-\int_{\partial_{2} \Omega} F u_{0} \mathrm{~d} s \\
& =\int_{\Omega} \nabla u_{0} \cdot \widehat{\mathbf{q}} \mathrm{d} x-\frac{1}{2}\|\widehat{\mathbf{q}}\|_{*}^{2}-l\left(u_{0}\right)
\end{aligned}
$$

and here $\widehat{\mathbf{q}}$ must satisfy the relation

$$
\int_{\Omega} \nabla w \cdot \widehat{\mathbf{q}} \mathrm{d} x=l(w) \quad \forall w \in V_{0} .
$$

In other cases, the functional $\widehat{I}^{*}(\widehat{\mathbf{q}})=-\infty$. This, in fact, means that Problems $\mathscr{P}^{*}$ and $\widehat{\mathscr{P}}^{*}$ coincide and are reduced to the maximization of $\widehat{I}^{*}$ on the set $Q_{l}$ (i.e. $\left.\sup \mathscr{P}^{*}=\sup \widehat{\mathscr{P}}^{*}\right)$.

Again, we have $\widehat{L}(\widehat{u}, \widehat{\mathbf{p}})=\inf \widehat{\mathscr{P}}=\sup \widehat{\mathscr{P}}^{*}$, but $\sup \widehat{\mathscr{P}}^{*}=\sup \mathscr{P}^{*}=\inf \mathscr{P}$ [see (1.9)]. Thus, we infer that $\inf \widehat{\mathscr{P}}=\inf \mathscr{P}$.

The latter equality implies that the minimizer $u \in V_{0}+u_{0}$ of Problem $\mathscr{P}$ is also the minimizer of the functional $\widehat{J}$ on $\widehat{V}$. Analogously, if $\mathbf{p} \in Q_{l}$ is the maximizer of Problem $\mathscr{P}^{*}$, then

$$
\int_{\Omega} \nabla w \cdot \mathbf{p} \mathrm{d} x=\int_{\Omega} f w \mathrm{~d} x+\int_{\partial_{2} \Omega} F w \mathrm{~d} s \quad \forall w \in V_{0} .
$$

From here we immediately see that $\operatorname{div} \mathbf{p}+f=0$ a.e. in $\Omega$ and, hence,

$$
\int_{\Omega}(\nabla w \cdot \mathbf{p}+(\operatorname{div} \mathbf{p}) w) \mathrm{d} x=\int_{\partial_{2} \Omega} F w \mathrm{~d} s \quad \forall w \in V_{0}
$$

that is $\mathbf{p} \in \widehat{Q}_{F}$. Thus, we conclude that $\mathbf{p}$ is also the maximizer of Problem $\widehat{\mathscr{P}}^{*}$.

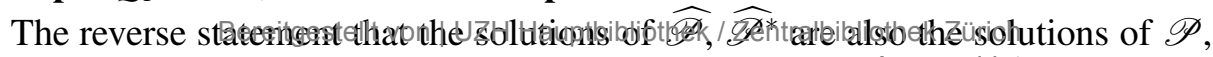
$\mathscr{P}^{*}$ is not difficult to prove either. Hence, both mixed formulations 
solution $(u, \mathbf{p})$ that is the generalized solution of problem (1.1)-(1.3).

If $\widehat{V}_{h} \subset \widehat{V}, \widehat{Q}_{0 h} \subset \widehat{Q}_{0}$ and $\widehat{Q}_{F h} \subset \widehat{Q}_{F}$ are certain finite element subspaces, then the discrete analog of (1.14)-(1.15) is the dual mixed finite element method (see, e.g. [6]): Find $\left(\widehat{u}_{h}, \widehat{\mathbf{p}}_{h}\right) \in \widehat{V}_{h} \times \widehat{Q}_{F h}$ such that

$$
\begin{gathered}
\int_{\Omega}\left(\mathbf{A}^{-1} \widehat{\mathbf{p}}_{h} \cdot \widehat{\mathbf{q}}_{h}+\left(\operatorname{div} \widehat{\mathbf{q}}_{h}\right) \widehat{u}_{h}\right) \mathrm{d} x=g\left(u_{0}, \widehat{\mathbf{q}}_{h}\right) \quad \forall \widehat{\mathbf{q}}_{h} \in \widehat{Q}_{0 h} \\
\int_{\Omega}\left(\operatorname{div} \widehat{\mathbf{p}}_{h}+f\right) \widehat{v}_{h} \mathrm{~d} x=0 \quad \forall \widehat{v}_{h} \in \widehat{V}_{h} .
\end{gathered}
$$

Now, our aim is to obtain computable upper bounds for the quantities

$$
\left\|\nabla\left(u-u_{h}\right)\right\| \mid, \quad\left\|\mathbf{p}-\mathbf{p}_{h}\right\|_{*}, \quad\left\|\mathbf{p}-\widehat{\mathbf{p}}_{h}\right\|_{\text {div }} .
$$

\section{A POSTERIORI ESTIMATES FOR THE PRIMAL MIXED FORMULATION}

First, we recall the relation (see, e.g. [17])

$$
\|\mathbf{p}-\mathbf{q}\|_{*}^{2}+\|\nabla(u-v)\|^{2}=2\left(J(v)-I^{*}(\mathbf{q})\right)
$$

where $\mathbf{q} \in Q_{l}$ and $v \in V_{0}+u_{0}$.

\section{Lemma 2.1.}

$$
J(v)-I^{*}(\mathbf{q}) \leqslant(1+\beta) D(\nabla v, \mathbf{y})+\frac{1}{2}\left(1+\frac{1}{\beta}\right)\|\mathbf{y}-\mathbf{q}\|_{*}^{2}
$$

where the functional $D$ was defined in (1.5), $v$ is an arbitrary function from $V_{0}+u_{0}$, $\mathbf{q}$ is an arbitrary function from $Q_{l}, \mathbf{y}$ is an arbitrary function from $Q$, and $\beta$ is any positive number.

Proof. We have

$$
\begin{aligned}
& J(v)-I^{*}(\mathbf{q})=\frac{1}{2} \mid\|\nabla v\|^{2}-l(v)+\frac{1}{2}\|\mathbf{q}\|_{*}^{2}+l\left(u_{0}\right)-\int_{\Omega} \nabla u_{0} \cdot \mathbf{q} \mathrm{d} x \\
& =\int_{\Omega}\left(\frac{1}{2} \mathbf{A} \nabla v \cdot \nabla v+\frac{1}{2} \mathbf{A}^{-1} \mathbf{y} \cdot \mathbf{y}-\nabla v \cdot \mathbf{y}\right) \mathrm{d} x+\int_{\Omega} \nabla\left(v-u_{0}\right) \cdot \mathbf{q} \mathrm{d} x \\
& \text { Bereitgestellt von I } \mathrm{NZH} \text { Hauptbiqliothek / Zentrapbibliothek Zürich }
\end{aligned}
$$

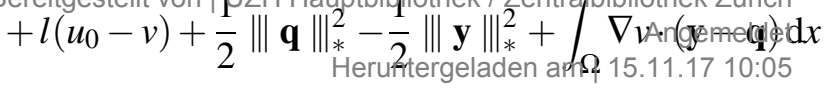




$$
\begin{aligned}
= & D(\nabla v, \mathbf{y})+\frac{1}{2}\|\mathbf{y}-\mathbf{q}\|_{*}^{2}-\int_{\Omega} \mathbf{A}^{-1} \mathbf{y} \cdot \mathbf{y} \mathrm{d} x+\int_{\Omega} \mathbf{A}^{-1} \mathbf{y} \cdot \mathbf{q} \mathrm{d} x \\
& +\int_{\Omega} \nabla v \cdot(\mathbf{y}-\mathbf{q}) \mathrm{d} x \\
= & D(\nabla v, \mathbf{y})+\frac{1}{2}\|\mathbf{y}-\mathbf{q}\|_{*}^{2}+\int_{\Omega}\left(\mathbf{A}^{-1} \mathbf{y}-\nabla v\right) \cdot(\mathbf{q}-\mathbf{y}) \mathrm{d} x .
\end{aligned}
$$

Since

$$
\int_{\Omega} \eta_{1} \cdot \eta_{2} \mathrm{~d} x \leqslant\left(\int_{\Omega} \mathbf{A} \eta_{1} \cdot \eta_{1} \mathrm{~d} x\right)^{1 / 2}\left(\int_{\Omega} \mathbf{A}^{-1} \eta_{2} \cdot \eta_{2} \mathrm{~d} x\right)^{1 / 2}
$$

we obtain

$$
\begin{aligned}
\int_{\Omega}\left(\mathbf{A}^{-1} \mathbf{y}-\nabla v\right) \cdot(\mathbf{q}-\mathbf{y}) \mathrm{d} x \leqslant & \left(\int_{\Omega} \mathbf{A}\left(\mathbf{A}^{-1} \mathbf{y}-\nabla v\right) \cdot\left(\mathbf{A}^{-1} \mathbf{y}-\nabla v\right) \mathrm{d} x\right)^{1 / 2} \\
& \times\left(\int_{\Omega} \mathbf{A}^{-1}(\mathbf{q}-\mathbf{y}) \cdot(\mathbf{q}-\mathbf{y}) \mathrm{d} x\right)^{1 / 2} \\
= & (2 D(\nabla v, \mathbf{y}))^{1 / 2}\|\mathbf{q}-\mathbf{y}\|_{*} \\
\leqslant & \beta D(\nabla v, \mathbf{y})+\frac{1}{2 \beta}\|\mathbf{q}-\mathbf{y}\|_{*}^{2}
\end{aligned}
$$

with an arbitrary positive number $\beta$, which immediately implies the estimate (2.2).

Lemma 2.2. Let $\mathbf{y} \in \widehat{Q}^{+}$. Then the following estimate holds true:

$$
\inf _{\mathbf{q} \in Q_{l}}\|\mathbf{q}-\mathbf{y}\|_{*}^{2} \leqslant C^{2}\left(\|\operatorname{div} \mathbf{y}+f\|^{2}+\|\mathbf{y} \cdot \mathbf{n}-F\|_{\partial_{2} \Omega}^{2}\right)
$$

where $C$ is the constant in the inequality

$$
\|w\|^{2}+\|w\|_{\partial_{2} \Omega}^{2} \leqslant C^{2}\|\nabla w\|^{2} \quad \forall w \in V_{0} .
$$

Proof. One can write

$$
\inf _{\mathbf{q} \in Q_{l}} \frac{1}{2}\|\mathbf{q}-\mathbf{y}\|_{*}^{2}=\inf _{\eta \in \widetilde{Q}_{l}} \frac{1}{2}\|\| \eta \|_{*}^{2}
$$

where $\widetilde{Q}_{l}:=\left\{\eta \in Q \mid \int_{\Omega} \nabla w \cdot \eta \mathrm{d} x=\int_{\Omega} \widetilde{f} w \mathrm{~d} x+\int_{\partial_{2} \Omega} \widetilde{F} w \mathrm{~d} s \forall w \in V_{0}\right\}$ with the notation $\widetilde{f}=f+\operatorname{div} \mathbf{y}$ and $\widetilde{F}=F-\mathbf{y} \cdot \mathbf{n}$.

Note that under the assumptions made we have $\widetilde{f} \in L_{2}(\Omega)$ and $\widetilde{F} \in L_{2}\left(\partial_{2} \Omega\right)$.

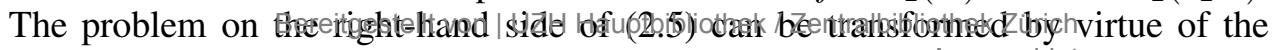
duality relations (see (1.8)), namely 


$$
\inf _{\eta \in \widetilde{Q}_{l}} \frac{1}{2}\|\| \|_{*}^{2}=-\sup _{\eta \in \widetilde{Q}_{l}}\left\{-\frac{1}{2}\|\eta\|_{*}^{2}\right\}=-\inf _{w \in V_{0}}\left\{\frac{1}{2}\|\nabla w\|^{2}-\widetilde{l}(w)\right\}
$$

where $\widetilde{l}(w)=\int_{\Omega} \widetilde{f} w \mathrm{~d} x+\int_{\partial_{2} \Omega} \widetilde{F} w \mathrm{~d} s$.

Further, we use inequality (2.4), where the constant $C$ depends only on the geometry of $\Omega$ and $\partial_{2} \Omega$ and on the bounds of the matrix $\mathbf{A}$ (see (1.4)). In fact, the constant can be presented as a combination of the constants from Friedrichs' and trace inequalities (see [19] for the discussion on these constants and ways of their evaluation).

Since

$$
\begin{aligned}
\widetilde{l}(w) & \leqslant\|\widetilde{f}\|\|w\|+\|\widetilde{F}\|_{\partial_{2} \Omega}\|w\|_{\partial_{2} \Omega} \leqslant\left(\|w\|^{2}+\|w\|_{\partial_{2} \Omega}^{2}\right)^{1 / 2}\left(\|\widetilde{f}\|^{2}+\|\widetilde{F}\|_{\partial_{2} \Omega}^{2}\right)^{1 / 2} \\
& \leqslant C\|\nabla w\|\left(\|\widetilde{f}\|^{2}+\|\widetilde{F}\|_{\partial_{2} \Omega}^{2}\right)^{1 / 2}
\end{aligned}
$$

we find that

$$
\begin{aligned}
\inf _{w \in V_{0}}\left\{\frac{1}{2}\|\nabla w\|^{2}-\widetilde{l}(w)\right\} & \geqslant \inf _{w \in V_{0}}\left\{\frac{1}{2}\|\nabla w\|^{2}-C\|\nabla w\|\left(\|\widetilde{f}\|^{2}+\|\widetilde{F}\|_{\partial_{2} \Omega}^{2}\right)^{1 / 2}\right\} \\
& \geqslant-\frac{1}{2} C^{2}\left(\|\widetilde{f}\|^{2}+\|\widetilde{F}\|_{\partial_{2} \Omega}^{2}\right) .
\end{aligned}
$$

Therefore,

$$
\inf _{\eta \in \widetilde{Q}_{l}} \frac{1}{2}\|\eta\|_{*}^{2} \leqslant \frac{C^{2}}{2}\left(\|\widetilde{f}\|^{2}+\|\widetilde{F}\|_{\partial_{2} \Omega}^{2}\right)
$$

which yields estimate (2.3).

Combining formula (2.1) with the results of Lemmas 2.1 and 2.2, we arrive at the following estimate:

$$
\begin{aligned}
& \|\mathbf{p}-\mathbf{q}\|_{*}^{2}+\|\nabla(u-v)\|^{2} \\
& \quad \leqslant 2(1+\beta) D(\nabla v, \mathbf{y})+\left(1+\frac{1}{\beta}\right) C^{2}\left(\|\operatorname{div} \mathbf{y}+f\|^{2}+\|\mathbf{y} \cdot \mathbf{n}-F\|_{\partial_{2} \Omega}^{2}\right)
\end{aligned}
$$

where $\mathbf{y} \in \widehat{Q}^{+}, \mathbf{q} \in Q_{l}$ and $v \in V_{0}+u_{0}$ are arbitrary functions and $\beta$ is any positive number. Thus, we have the general estimate for the error in the primal variable:

$$
\begin{aligned}
& \|\nabla(u-v)\|^{2} \leqslant 2(1+\beta) D(\nabla v, \mathbf{y})
\end{aligned}
$$

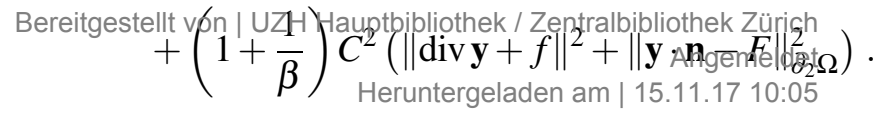


It is worth noting that for any 'approximate solution' $v \in V_{0}+u_{0}$ the estimate is exact in the sense that there is a function $\mathbf{y} \in \widehat{Q}^{+}$, (namely, $\mathbf{y}=\mathbf{A} \nabla u$ ) such that the inequality becomes an equality (the last two terms will then be zero and $\beta$ can be taken arbitrarily small).

Since $u_{h} \in V_{0}+u_{0}$, we can substitute it into (2.8) instead of $v$ and obtain an upper bound for $\left\|\nabla\left(u-u_{h}\right)\right\|^{2}$; however, the 'free function' $\mathbf{y} \in \widehat{Q}^{+}$is still to be chosen. There are several possibilities to find a good approximation of the exact flux $\mathbf{A} \nabla u$ i $\widehat{Q}^{+}$, i.e., a candidate for the role of $\mathbf{y}$ in (2.8). The cheapest way is to use the function $\mathbf{p}_{h} \in Q_{h}$ available from the solution of the primal mixed problem and to construct the projection operator $\mathscr{D}_{h}: Q_{h} \rightarrow \widehat{Q}^{+}$, so that $\mathscr{D}_{h} p_{h}$ could be inserted into (2.8) in place of $\mathbf{y}$. This projection as well as other methods for finding $\mathbf{y} \in \widehat{Q}^{+}$are discussed below.

Now, we derive an estimate for the error in the dual variable. If $\mathbf{p}_{h}$ were in $Q_{l}$, we could directly apply (2.7) to obtain an upper bound for $\left\|\mathbf{p}-\mathbf{p}_{h}\right\|_{*}^{2}$. However, this is not the case, as the function $\mathbf{p}_{h}$ satisfies the relation

$$
\int_{\Omega} \mathbf{p}_{h} \cdot \nabla w_{h} \mathrm{~d} x=l\left(w_{h}\right) \quad \forall w_{h} \in V_{0 h} \subset V_{0}
$$

which defines a set of vector-valued functions wider than $Q_{l}$. This difficulty is circumvented as follows.

Let $\mathbf{y} \in \widehat{Q}^{+}$and $\mathbf{q} \in Q_{l}$. Then

$$
\begin{aligned}
\left\|\mid \mathbf{p}-\mathbf{p}_{h}\right\|_{*} & \leqslant\|\mathbf{p}-\mathbf{q}\|_{*}+\|\mathbf{q}-\mathbf{y}\|_{*}+\left\|\mathbf{y}-\mathbf{p}_{h}\right\|_{*} \\
& \leqslant \sqrt{2}\left(J(v)-I^{*}(\mathbf{q})\right)^{1 / 2}+\|\mathbf{q}-\mathbf{y}\|_{*}+\left\|\mathbf{y}-\mathbf{p}_{h}\right\|_{*} .
\end{aligned}
$$

By Lemma 2.1 (after minimizing the right-hand side of (2.2) with respect to the positive scalar parameter $\beta$ )

$$
\sqrt{2}\left(J(v)-I^{*}(\mathbf{q})\right)^{1 / 2} \leqslant \sqrt{2} D^{1 / 2}(\nabla v, \mathbf{y})+\|\mathbf{q}-\mathbf{y}\|_{*} .
$$

Thus,

$$
\left\|\mathbf{p}-\mathbf{p}_{h}\right\|_{*} \leqslant \sqrt{2} D^{1 / 2}(\nabla v, \mathbf{y})+2\|\mathbf{q}-\mathbf{y}\|_{*}+\left\|\mathbf{y}-\mathbf{p}_{h}\right\|_{*}
$$

and, taking the infimum of the right-hand side over all $\mathbf{q} \in Q_{l}$ and using Lemma 2.2, one obtains

$$
\begin{aligned}
\left\|\mathbf{p}-\mathbf{p}_{h}\right\|_{*} \leqslant & \sqrt{2} D^{1 / 2}(\nabla v, \mathbf{y})+\left\|\mathbf{y}-\mathbf{p}_{h}\right\|_{*} \\
& +2 C\left(\|\operatorname{div} \mathbf{y}+f\|^{2}+\|\mathbf{y} \cdot \mathbf{n}-F\|_{\partial_{2} \Omega}^{2}\right)^{1 / 2} .
\end{aligned}
$$

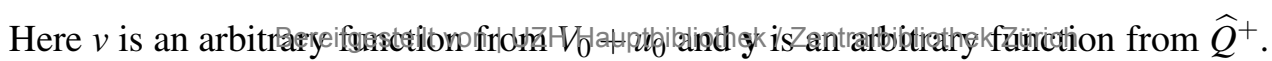
Note that, if $\mathbf{y}=\mathbf{A} \nabla u$ and $v=u$, the right-hand side of (2. A pyerointetdes with the 
left-hand side, i.e., estimate (2.11) is exact in the sense that one can always find 'free variables' in such a way that the inequality becomes an equality.

A directly computable upper bound of $\left\|\mathbf{p}-\mathbf{p}_{h}\right\|_{*}$ is given by (2.11), if we set $v=u_{h}$ and $\mathbf{y}=\mathscr{D}_{h} p_{h}$, where $\mathscr{D}_{h}: Q_{h} \rightarrow \widehat{Q}^{+}$is the projection operator mentioned above. It is clear that for obtaining the computable bounds of errors in both the primal and dual variables, we need to construct the projection of $\mathbf{p}_{h}$ onto the space $\widehat{Q}^{+}$. This can be easily done, for instance, by simple averaging, as we are about to see.

\subsection{Projection from $Q_{h}$ onto $\widehat{Q}^{+}$}

If $\mathbf{p}_{h}$ is a piecewise-constant vector field on a simplicial mesh $\mathscr{T}_{h}$, we can readily project it onto the subspace of $\widehat{Q}^{+}$that is formed by the Raviart-Thomas elements of the lowest order $\mathbf{R T}_{0}$ (see $[6,15]$ ). Below, we consider a two-dimensional case for the sake of simplicity, but the extension to the three-dimensional case is straightforward. We also assume that the domain $\Omega$ has a polygonal boundary and the latter is exactly matched by the triangulation $\mathscr{T}_{h}$.

Let $T_{i}$ and $T_{j}$ be two neighbouring simplexes with the common edge $E_{i j}$. If $\mathbf{q}_{h}$ is a piecewise constant vector-valued function that has the values $\mathbf{q}_{i}$ and $\mathbf{q}_{j}$ on $T_{i}$ and $T_{j}$, respectively, we set on $E_{i j}$ the value of the normal component of the 'averaged flux' as follows:

$$
\widetilde{\mathbf{q}}_{i j} \cdot \mathbf{n}_{i j}=\frac{1}{2}\left(\mathbf{q}_{i}+\mathbf{q}_{j}\right) \cdot \mathbf{n}_{i j}
$$

where $\mathbf{n}_{i j}$ is the unit normal to the edge $E_{i j}$ oriented from $T_{i}$ to $T_{j}$ if $i>j$. Another option is

$$
\widetilde{\mathbf{q}}_{i j} \cdot \mathbf{n}_{i j}=\frac{\left|T_{i}\right| \mathbf{q}_{i}+\left|T_{j}\right| \mathbf{q}_{j}}{\left|T_{i}\right|+\left|T_{j}\right|} \cdot \mathbf{n}_{i j}
$$

where $\left|T_{i}\right|$ and $\left|T_{j}\right|$ are the areas of $T_{i}$ and $T_{j}$. We repeat this procedure for all internal edges of $\mathscr{T}_{h}$.

If $E_{i 0} \in \partial_{1} \Omega$, then we set $\widetilde{\mathbf{q}}_{i 0} \cdot \mathbf{n}_{i 0}=\mathbf{q}_{i 0} \cdot \mathbf{n}_{i 0}$. If $E_{i 0} \in \partial_{2} \Omega$, then

$$
\widetilde{\mathbf{q}}_{i 0} \cdot \mathbf{n}_{i 0}=\frac{1}{\left|E_{i 0}\right|} \int_{E_{i 0}} F \mathrm{~d} s .
$$

Here $\left|E_{i 0}\right|$ is the length of the edge $E_{i 0}$.

Thus, all normal components $\widetilde{\mathbf{q}}_{i j} \cdot \mathbf{n}_{i j}$ on internal and external edges are defined. By prolongation inside all $T_{i}, i=1, \ldots, N$, with the help of $\mathbf{R} \mathbf{T}_{0}$-approximation we obtain the function

$$
\widetilde{\mathbf{q}}_{h}=\mathscr{D}_{h} \mathbf{q}_{h} \in \widehat{Q}^{+} .
$$

We are now ready to show a posteriori estimates for the errors in the primal and the dual variables for the primal mixed finite element method.

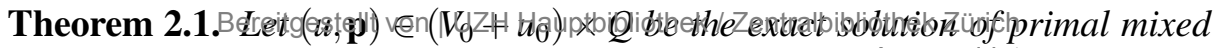
problem (1.6)-(1.7) and $\left(u_{h}, \mathbf{p}_{h}\right) \in\left(V_{0 h}+u_{0}\right) \times Q_{h}$ the solutibremplalis crete primal 
mixed problem (1.10)-(1.11).

Then, the following a posteriori estimates hold true:

$$
\begin{aligned}
\|\| \nabla\left(u-u_{h}\right) \| \leqslant & \left\|\mathbf{A} \nabla u_{h}-\mathscr{D}_{h} \mathbf{p}_{h}\right\|_{*} \\
& +C\left(\left\|\operatorname{div}\left(\mathscr{D}_{h} \mathbf{p}_{h}\right)+f\right\|^{2}+\left\|\left(\mathscr{D}_{h} \mathbf{p}_{h}\right) \cdot \mathbf{n}-F\right\|_{\partial_{2} \Omega}^{2}\right)^{1 / 2} \\
\left\|\mathbf{p}-\mathbf{p}_{h}\right\|_{*} \leqslant & \left\|\mathbf{A} \nabla u_{h}-\mathscr{D}_{h} \mathbf{p}_{h}\right\|_{*}+\left\|\mathscr{D}_{h} \mathbf{p}_{h}-\mathbf{p}_{h}\right\| \|_{*} \\
& +2 C\left(\left\|\operatorname{div}\left(\mathscr{D}_{h} \mathbf{p}_{h}\right)+f\right\|^{2}+\left\|\left(\mathscr{D}_{h} \mathbf{p}_{h}\right) \cdot \mathbf{n}-F\right\|_{\partial_{2} \Omega}^{2}\right)^{1 / 2}
\end{aligned}
$$

where $\mathscr{D}_{h}: Q_{h} \rightarrow \widehat{Q}^{+}$is the projection (averaging) operator introduced above and $C$ is the constant from Lemma 2.2.

Proof. The first estimate immediately follows from (2.8) if we set there $v=u_{h}$, $\mathbf{y}=\mathscr{D}_{h} \mathbf{p}_{h}$, use definition (1.5) of the functional $D$ and then minimize the right-hand side of (2.8) with respect to the scalar parameter $\beta$.

Estimate (2.13) is a direct consequence of (2.11).

Remark 2.1. The first term in estimates (2.12), (2.13) computed elementwise can serve as a local error indicator (see also $[18,19])$.

Remark 2.2. A better estimate for $\left\|\nabla\left(u-u_{h}\right)\right\| \mid$ can be derived by minimizing the right-hand side of (2.8) with respect to $\beta>0$ and $\mathbf{y} \in \widehat{Q}^{+}$. As to $\mathbf{y}$, one has to minimize over some finite-dimensional subspace $\widehat{Q}_{h}^{+} \subset \widehat{Q}^{+}$, for instance, the Raviart-Thomas space $\mathbf{R} \mathbf{T}_{0}$. A detailed discussion on the minimization of the functional of form (2.8) can be found in [18].

\section{A POSTERIORI ESTIMATES FOR DUAL MIXED FORMULATION}

The a posteriori estimate for the flux $\widehat{\mathbf{p}}_{h}$ readily follows from (2.11) if we set $\mathbf{y}=$ $\widehat{\mathbf{p}}_{h} \in \widehat{Q}^{+}$:

$$
\left\|\mathbf{p}-\widehat{\mathbf{p}}_{h}\right\|_{*} \leqslant \sqrt{2} D^{1 / 2}\left(\nabla v, \widehat{\mathbf{p}}_{h}\right)+2 C\left(\left\|\operatorname{div} \widehat{\mathbf{p}}_{h}+f\right\|^{2}+\left\|\widehat{\mathbf{p}}_{h} \cdot \mathbf{n}-F\right\|_{\partial_{2} \Omega}^{2}\right)^{1 / 2}
$$

where $v$ is an arbitrary function from $V_{0}+u_{0}$.

Suppose that $\Omega$ is a polygonal domain whose boundary is exactly resolved by the triangulation $\mathscr{T}_{h}$. If $\widehat{\mathbf{p}}_{h}$ is constructed by means of $\mathbf{R T}_{0}$-elements, then

$$
\int_{\Omega}\left(\operatorname{div} \widehat{\mathbf{p}}_{h}+f\right) w_{h} \mathrm{~d} x=0 \quad \forall w_{h} \in \widehat{V}_{h} \subset \widehat{V}
$$

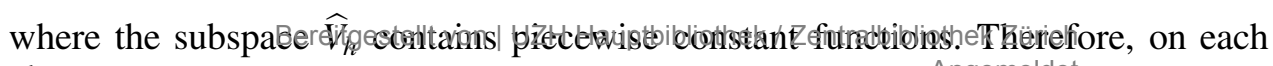
element $T_{i}$ 


$$
\operatorname{div} \widehat{\mathbf{p}}_{h}=-\frac{1}{\left|T_{i}\right|} \int_{T_{i}} f \mathrm{~d} x
$$

Let us define by $[f]$ the function that belongs to $\widehat{V}_{h}$ and whose values on $T_{i}$ coincide with the mean values of $f$ on $T_{i}$. Then we have $\operatorname{div} \widehat{\mathbf{p}}_{h}=-[f]$ on every $T_{i}$.

We now see that estimate (3.1) is valid for any approximate flux $\widehat{\mathbf{p}}_{h}$ from $\widehat{Q}^{+}$. If $\widehat{\mathbf{p}}_{h}$ were in the narrower set $\widehat{Q}_{F}$ [as is supposed to be the case in the discrete dual mixed method (1.22)-(1.23)], the last norm in (3.1) would be identical to zero. It cannot, however, be expected when $\widehat{\mathbf{p}}_{h}$ is constructed in the space $\mathbf{R} \mathbf{T}_{0}$, unless the function $F$ is a constant on $\partial_{2} \Omega$.

The problem of taking into account the essential boundary condition for the flux variable $\left(\widehat{\mathbf{p}} \cdot \mathbf{n}=F\right.$ on $\partial_{2} \Omega$ ) in the dual mixed method is not easy and usually leads to a nonconforming approximation $\widehat{\mathbf{p}}_{h}$ (see, e.g. [3] for the use of the Lagrange multiplier technique in this case). Since our estimate (3.1) still works for these approximations of the flux, we propose a simple modification of the discrete dual method (1.22)-(1.23) particularly suited for the lowest-order Raviart-Thomas approximation.

Namely, instead of requiring $\widehat{\mathbf{p}}_{h} \in \widehat{Q}_{F}$, we impose a weaker condition

$$
\left.\widehat{\mathbf{p}}_{h} \cdot \mathbf{n}\right|_{E_{i 0}}=\frac{1}{\left|E_{i 0}\right|} \int_{E_{i 0}} F \mathrm{~d} s
$$

on every edge $E_{i 0} \in \partial_{2} \Omega$. The space of test functions $\widehat{Q}_{0 h} \subset \widehat{Q}_{0}$ will obviously consist of $\mathbf{R} \mathbf{T}_{0}$-approximations $\widehat{\mathbf{q}}_{h}$ such that $\widehat{\mathbf{q}}_{h} \cdot \mathbf{n}=0$ on each edge $E_{i 0} \in \partial_{2} \Omega$.

If we now denote by $[F]$ the piecewise constant function defined on the set of edges forming $\partial_{2} \Omega$ and whose value on every edge $E_{i 0} \in \partial_{2} \Omega$ is equal to the mean value of $F$ on that edge, we can write $\widehat{\mathbf{p}}_{h} \cdot \mathbf{n}=[F]$ for all $E_{i 0} \in \partial_{2} \Omega$.

As a result, we obtain from (3.1)

$$
\left\|\mathbf{p}-\widehat{\mathbf{p}}_{h}\right\|_{*} \leqslant \sqrt{2} D^{1 / 2}\left(\nabla v, \widehat{\mathbf{p}}_{h}\right)+2 C\left(\|f-[f]\|^{2}+\|F-[F]\|_{\partial_{2} \Omega}^{2}\right)^{1 / 2} .
$$

The question that now arises is how to choose in this inequality the function $v \in$ $V_{0}+u_{0}$. The simplest way is to use the function $\widehat{u}_{h} \in \widehat{V}_{h}$ available from the solution of the discrete dual mixed problem and to construct a suitable projection operator $\mathscr{P}_{h}: \widehat{V}_{h} \rightarrow V_{0}+u_{0}$. Again, the projection can be easily accomplished by simple averaging.

\subsection{Projection from $\widehat{V}_{h}$ onto $V_{0}+u_{0}$}

In order to find $v \in V_{0}+u_{0}$, it is sufficient to find $w \in V_{0}$ in the representation $v=$ $w+u_{0}$ (the function $u_{0}$ is given). Using the computed piecewise-constant function $\widehat{u}_{h}$, we define $w_{h}$ eEtgitas ffellows. UZH Hauptbibliothek / Zentralbibliothek Zürich

We set 


$$
w_{h}\left(x_{k}\right)=\frac{\left.\sum_{s=1}^{N_{k}}\left|T_{s}^{(k)}\right| \cdot \widehat{u}_{h}\right|_{T_{s}^{(k)}}}{\sum_{s=1}^{N_{k}}\left|T_{s}^{(k)}\right|}-u_{0}\left(x_{k}\right)
$$

for any internal node $x_{k}$ and when $x_{k} \in \partial_{2} \Omega$. Here $T_{s}^{(k)}, s=1, \ldots, N_{k}$, are the elements containing the vertex $x_{k}$, and we have assumed that the function $u_{0}$ has a sufficient regularity so that its point values are defined.

If the node $x_{k} \in \partial_{1} \Omega$, we simply set $w_{h}\left(x_{k}\right)=0$.

Thus, using the nodal values of $w_{h}$ and the piecewise-linear continuous finite element approximation on the mesh $\mathscr{T}_{h}$, we define the function

$$
w_{h}+u_{0}=\mathscr{P}_{h} \widehat{u}_{h} \in V_{0}+u_{0} .
$$

Hence, from (3.5) one obtains

$$
\left\|\mathbf{p}-\widehat{\mathbf{p}}_{h}\right\|_{*} \leqslant \sqrt{2} D^{1 / 2}\left(\nabla\left(\mathscr{P}_{h} \widehat{u}_{h}\right), \widehat{\mathbf{p}}_{h}\right)+2 C\left(\|f-[f]\|^{2}+\|F-[F]\|_{\partial_{2} \Omega}^{2}\right)^{1 / 2}
$$

which, together with the obvious relation

$$
\left\|\operatorname{div}\left(\widehat{\mathbf{p}}-\widehat{\mathbf{p}}_{h}\right)\right\|=\left\|-f-\operatorname{div} \widehat{\mathbf{p}}_{h}\right\|=\|f-[f]\|
$$

yields the following upper bound for $\left\|\widehat{\mathbf{p}}-\widehat{\mathbf{p}}_{h}\right\|_{\text {div }}$.

Theorem 3.1. Let $(\widehat{u}, \widehat{\mathbf{p}}) \in \widehat{V} \times \widehat{Q}_{F}$ be the exact solution of dual mixed problem (1.14)-(1.15) and $\left(\widehat{u}_{h}, \widehat{\mathbf{p}}_{h}\right) \in \widehat{V}_{h} \times \widehat{Q}_{F h}$ the solution of discrete dual mixed problem (1.22)-(1.23) with $\widehat{Q}_{F h}$ being the Raviart-Thomas space $\mathbf{R T}_{0}$ equipped with conditions (3.4). Then, the following estimate holds true:

$$
\left\|\widehat{\mathbf{p}}-\widehat{\mathbf{p}}_{h}\right\|_{\text {div }} \leqslant\left\|\mathbf{A} \nabla\left(\mathscr{P}_{h} \widehat{u}_{h}\right)-\widehat{\mathbf{p}}_{h}\right\|_{*}+(2 C+1)\|f-[f]\|+2 C\|F-[F]\|_{\partial_{2} \Omega}
$$

where $\mathscr{P}_{h}: \widehat{V}_{h} \rightarrow V_{0}+u_{0}$ is the projection (averaging) operator introduced above, $C$ is the constant from Lemma 2.2, $[f]$ and $[F]$ are the averaged functions (see the right-hand sides of (3.3) and (3.4)).

Remark 3.1. The first and the second terms in (3.9) computed elementwise can be used as local indicators for the $L_{2}$-error and the error in the equation $\operatorname{div} \widehat{\mathbf{p}}+f=0$, respectively.

Remark 3.2. A possibly sharper estimate may be derived if one has the conforming finite elemeatgGalerkin sohution, toierigimal problemidthek)z(1, $B)$ and inserts it in (3.9) instead of $\mathscr{P}_{h} \widehat{u}_{h}$. 
Remark 3.3. Yet a better estimate may be derived by minimizing the right-hand side of (3.5) with respect to $v$. Here, we can restrict ourselves to the minimization of the functional $D\left(\nabla v, \widehat{\mathbf{p}}_{h}\right)$ over the set $V_{0 h}+u_{0}$, i.e., to the finite-dimensional problem

$$
\min _{w_{h} \in V_{0 h}}\left(\int_{\Omega}\left(\frac{1}{2} \mathbf{A} \nabla w_{h} \cdot \nabla w_{h}+\left(\mathbf{A} \nabla u_{0}-\widehat{\mathbf{p}}_{h}\right) \cdot \nabla w_{h}\right) \mathrm{d} x\right) .
$$

Under the assumption on the proper regularity of $u_{0}$, this problem is transformed as follows

$$
\min _{w_{h} \in V_{0 h}}\left(\int_{\Omega}\left(\frac{1}{2} \mathbf{A} \nabla w_{h} \cdot \nabla w_{h}-f_{0} w_{h}\right) \mathrm{d} x-\int_{\partial_{2} \Omega} F_{0} w_{h} \mathrm{~d} s\right)
$$

where $f_{0}=\operatorname{div}\left(\mathbf{A} \nabla u_{0}\right)+[f]$ and $F_{0}=[F]-\mathbf{A} \nabla u_{0} \cdot \mathbf{n}$. In the particular case $u_{0}=0$, the best choice of $w_{h}$ is to take it as the Galerkin approximation of the original problem (1.1)-(1.3) with averaged right-hand sides $[f]$ and $[F]$.

Although the primal variable $\widehat{u}$ is sought in the space $L_{2}(\Omega)$ in the framework of the dual mixed method, the exact solution $u$ of problem (1.1)-(1.3) belongs to the space $V_{0}+u_{0} \subset H^{1}(\Omega)$. Therefore, it does not seem unnatural to measure the error of the primal variable approximation in the energy norm $\||\nabla \cdot|\|$ in the dual mixed approach as well. In fact, with estimate (2.8), we can assess the squared norm of the error of the averaged solution $\mathscr{P}_{h} \widehat{u}_{h}$ using the computed flux approximation $\widehat{\mathbf{p}}_{h}$ :

$$
\begin{aligned}
\left\|\nabla\left(u-\mathscr{P}_{h} \widehat{u}_{h}\right)\right\|^{2} \leqslant & 2(1+\beta) D\left(\nabla\left(\mathscr{P}_{h} \widehat{u}_{h}\right), \widehat{\mathbf{p}}_{h}\right) \\
& +\left(1+\frac{1}{\beta}\right) C^{2}\left(\|f-[f]\|^{2}+\|F-[F]\|_{\partial_{2} \Omega}^{2}\right)
\end{aligned}
$$

where $\beta>0$ is an arbitrary number that can be used to minimize the right-hand side of (3.12) and derive an estimate for the norm of the error.

A sharper estimate may be derived if one spends some time on the minimization of the right-hand side of (2.8) with respect to the dual variable $\mathbf{y}$ over some finitedimensional subspace of $\widehat{Q}^{+}$.

Remark 3.4. If one has the solutions of both the primal and dual mixed problems, the flux approximation $\widehat{\mathbf{p}}_{h}$ can be substituted into (2.8) to immediately yield the error estimate for the primal variable (which is the most important in the primal mixed method), while the approximation $u_{h}$ can be used in (3.5) to derive the error estimate for the dual variable (which is the most important in the dual mixed method). 


\section{REFERENCES}

1. Y. Achdou, C. Bernardi, and F. Coquel, A priori and a posteriori analysis of finite volume discretizations of Darcy's equations. Numer. Math. (2003) 96, No. 1, 17-42.

2. A. Alonso, Error estimators for a mixed method. Numer. Math. (1996) 74, 385-395.

3. I. Babuška and G. N. Gatica, On the mixed finite element method with Lagrange multipliers. Numer. Meth. Partial Diff. Equations (2003) 19, No. 2, 192-210.

4. D. Braess and R. Verfürth, A posteriori error estimators for the Raviart-Thomas element. SIAM J. Numer. Anal. (1996) 33, No. 6, 2431-2444.

5. J. H. Brandts, Superconvergence and a posteriori error estimation for triangular mixed finite elements. Numer. Math. (1994) 68, No. 3, 311-324.

6. F. Brezzi and M. Fortin, Mixed and Hybrid Finite Element Methods. Springer-Verlag, New York, 1991.

7. C. Carstensen, A posteriori error estimate for the mixed finite element method. Math. Comp. (1997) 66, No. 218, 465-476.

8. C. Carstensen and S. Bartels, Each averaging technique yields reliable a posteriori error control in FEM on unstructured grids. I Low order conforming, non-conforming and mixed FEM. Math. Comp. (2002) 71, No. 239, 945-969.

9. C. Carstensen and G. Dolzmann, A posteriori error estimates for mixed FEM in elasticity. Numer. Math. (1998) 81, 187-209.

10. I. Ekeland and R. Temam, Convex Analysis and Variational Problems. North-Holland, New York, 1976.

11. G. Gatica and M. Maischak, A posteriori error estimates for the mixed finite element method with Lagrange multipliers. Numer. Meth. Partial Diff. Equations (to appear).

12. V. Girault and P.-A. Raviart, Finite Element Methods for Navier-Stokes Equations. Theory and Algorithms. Springer-Verlag, New York, 1986.

13. Yu. Kuznetsov and S. Repin, New mixed finite element method on polygonal and polyhedral meshes. Russian J. Numer. Anal. Math. Modelling (2003) 18, No. 3, 261-278.

14. M. Lonsing and R. Verfürth, A posteriori error estimators for mixed finite element methods in linear elasticity. Numer. Math. (2004) 97, 757-778.

15. P.-A. Raviart and J.-M. Thomas, A mixed finite element for second order elliptic problems. In: Mathematical Aspects of Finite Element Methods (Eds. I. Galligani and E. Magenes). SpringerVerlag, Berlin, 1977, pp. 292-315.

16. S. Repin, A posteriori error estimation for variational problems with uniformly convex functionals. Math. Comp. (2000) 69, No. 230, 481-500.

17. S. Repin, Two-sided estimates of deviation from exact solutions of uniformly elliptic equations. Amer. Math. Soc. Transl. Series 2 (2003) 209, 143-171.

18. S. Repin, S. Sauter, and A. Smolianski, A posteriori error estimation for the Dirichlet problem with account of the error in approximation of boundary conditions. Computing (2003) 70, No. 3, 205-233.

19. S. Repin, S. Sauter, and A. Smolianski, A posteriori error estimation for the Poisson equation with mixed Dirichlet-Neumann boundary conditions. J. Comp. Appl. Math. (2004) 164/165, 601-612.

20. J. E. Roberts and J.-M. Thomas, Mixed and hybrid methods. In: Handbook of Numerical Analy-

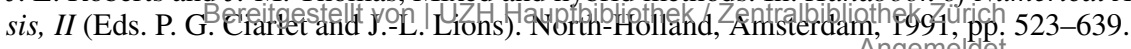


21. M. Vohralik, Equivalence between mixed finite element and multi-point finite volume methods. C. R. Acad. Sci. Paris, Ser. I (2004) 339, 525-528.

22. B. I. Wohlmuth and R. H. W. Hoppe, A comparison of a posteriori error estimators for mixed finite element discretizations by Raviart-Thomas elements. Math. Comp. (1999) 68, No. 228, 1347-1378.

23. A. Younes, P. Ackerer, and G. Chavent, From mixed finite elements to finite volumes for elliptic PDEs in two and three dimensions. Int. J. Numer. Meth. Engrg. (2004) 59, 365-388. 